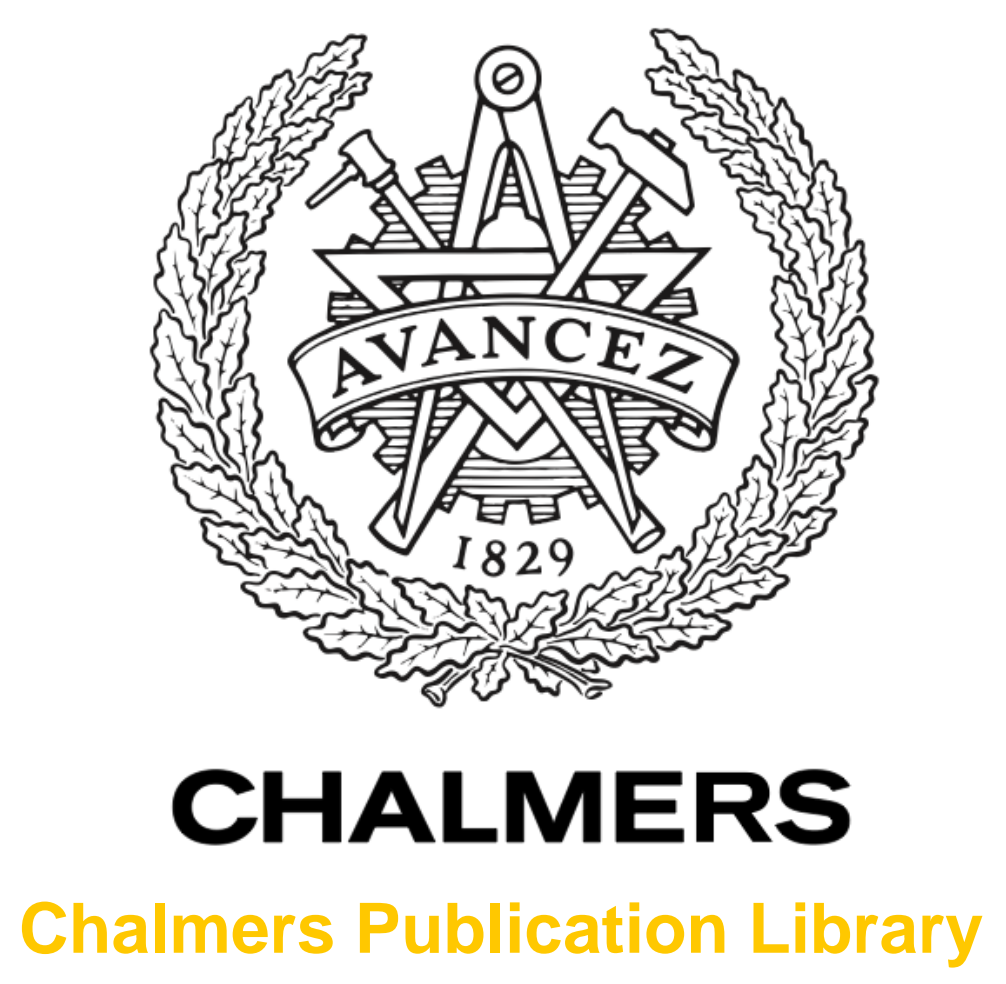

\title{
Thick barrier noise-reduction in the presence of atmospheric turbulence: Measurements and numerical modelling
}

This document has been downloaded from Chalmers Publication Library (CPL). It is the author's version of a work that was accepted for publication in:

Applied Acoustics (ISSN: 0003-682X)

Citation for the published paper:

Forssén, J. ; Ögren, M. (2002) "Thick barrier noise-reduction in the presence of atmospheric turbulence: Measurements and numerical modelling". Applied Acoustics, vol. 63(2), pp.

173-187.

http://dx.doi.org/10.1016/S0003-682X(01)00028-7

Downloaded from: http://publications.lib.chalmers.se/publication/23206

Notice: Changes introduced as a result of publishing processes such as copy-editing and formatting may not be reflected in this document. For a definitive version of this work, please refer to the published source. Please note that access to the published version might require a subscription.

Chalmers Publication Library (CPL) offers the possibility of retrieving research publications produced at Chalmers University of Technology. It covers all types of publications: articles, dissertations, licentiate theses, masters theses, conference papers, reports etc. Since 2006 it is the official tool for Chalmers official publication statistics. To ensure that Chalmers research results are disseminated as widely as possible, an Open Access Policy has been adopted.

The CPL service is administrated and maintained by Chalmers Library. 


\title{
Barrier noise-reduction in the presence of atmospheric turbulence: Measurements and numerical modelling
}

\begin{abstract}
Atmospheric turbulence causes scattering of sound, which can reduce the performance of sound barriers. This is important to include in prediction models to get a correct picture of the sound reduction at higher frequencies. Here a prediction method is applied that uses the strengths of the wind and temperature turbulence to estimate the scattered power into the shadow zone of a barrier. The predictions are compared to full-scale measurements on a thick barrier, where both acoustic and meteorological data were recorded simultaneously under both calm and windy conditions. Comparison between the measurements and the predictions indicate that the method gives reasonably accurate results for mid to high frequencies and a slight overestimation at very high frequencies.
\end{abstract}

Keywords: barrier, screen, atmospheric turbulence, scattering, decorrelation, measurements, predictions.

\section{Introduction}

When trying to predict the sound reduction obtained with a noise barrier in an outdoor environment, the inhomogeneous nature of the atmosphere can be of importance to consider. Wind speed and temperature that vary with height cause refraction and the atmospheric turbulence causes scattering and decorrelation of the sound waves. The turbulence scattering can significantly decrease the sound reduction obtained with a noise barrier, especially for high frequencies and large scale geometries [1, 2]. An example of a situation with a large scale geometry is a large building along a road side.

There exist other models than the one used here for predicting the influence of atmospheric turbulence on noise barrier performance. A parabolic equation method has been applied to situations with flat geometries [3], i.e. when the source and the receiver are far away from the barrier in relation to the height of 
the barrier. Also a model called the substitute-sources model has been developed [4]. In this model a surface of sources represents the field behind the barrier and a mutual coherence between all source-pairs is used which describes the decorrelation due to the turbulence. Also this model has so far only been implemented for flat geometries.

The model used here for predicting the effects of a turbulent atmosphere on the sound reduction by a barrier on ground is based on a model by Daigle [5] and has been further extended. In Daigle's model the power scattered by the turbulence is calculated using a sound scattering cross-section [6, 7] and is then added to the diffracted power in the acoustic shadow of the barrier. With this model Daigle investigated a few different geometries and the predictions were compared with measured data [5]. The comparison showed a fairly good agreement between predictions and measurements, and that neglecting the turbulence scattering would yield a poor prediction, especially at higher frequencies, which is also concluded in Ref. [8].

Using the physically based Kolmogorov spectrum for the description of the turbulence allows for a straight forward transformation of the results from one frequency to other frequencies. Moreover, the results when enlarging or diminishing the geometry in scale can also be predicted using straight forward transformations. These transformation properties are adopted and the predictions for many situations can be made from a small set of pre-calculated data, as shown in Refs. [1, 2], and as described in the following. In the further extended model used here, also the interference effects due to a ground surface are taken into account, in an approximate way.

\section{Measurement setup}

Two sets of measurements were carried out with identical measurement setups. One set of measurements was carried out during calm weather conditions with rather low wind speeds $(1-3 \mathrm{~m} / \mathrm{s})$ and weak turbulence. During the other set the wind speeds were higher $(4-7 \mathrm{~m} / \mathrm{s})$ and the turbulence was stronger. Unfortunately the wind direction had changed between the two sets, so during the calm set there was predominately upwind propagation, and downwind for the windier set.

The barrier was constructed using two standard containers, one $12 \mathrm{~m}$ and one $6 \mathrm{~m}$ long, forming an $18 \mathrm{~m}$ long barrier, $2.55 \mathrm{~m}$ high and $2.44 \mathrm{~m}$ thick. The containers were placed on a wide asphalt road strip, and mineral wool absorbing material was used to seal the gap between them. The source was positioned $8 \mathrm{~m}$ from the barrier during all measurements, and the receiving microphone 


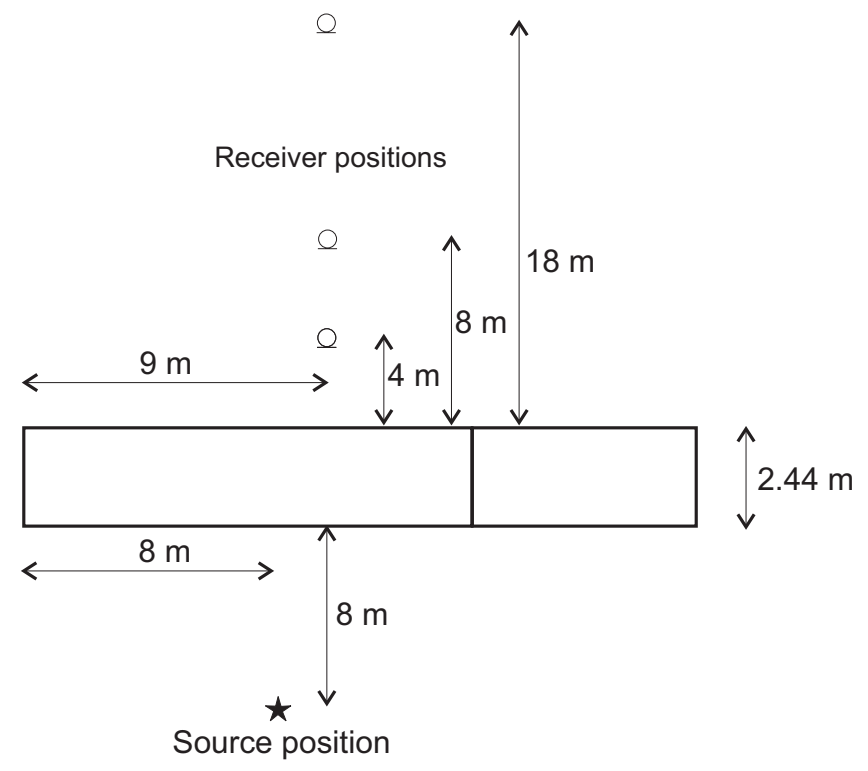

Figure 1. Measurement setup.

was placed at a distance of 4,8 or $18 \mathrm{~m}$ from the barrier. The geometry is described in detail in Figure 1. The microphone and the source were placed either on the ground or at a height of $1.25 \mathrm{~m}$ above ground.

A point source was simulated using a compression driver feeding into a flexible hose with a diameter of $25 \mathrm{~mm}$ at the opening. A CD-player supplied a filtered pink noise signal trough an amplifier to the driver. One $1 / 4^{\prime \prime}$ microphone was placed close to the source to monitor the emitted sound, and a second $1 / 2^{\prime \prime}$ microphone was used to measure the sound pressure at the receiving positions. The signals were recorded using a DAT recorder at a sampling frequency of $48 \mathrm{kHz}$, and were later transformed to power spectra using the Welch method with a Hanning window of length 2048 points and 50\% overlap.

The wind speed was measured using a conventional instrument with rotating cups. The sensor was placed far away from the barrier to avoid disturbances, at a height of $5 \mathrm{~m}$. The turbulence was measured using an ultrasound anemometer with a sampling frequency of $18 \mathrm{~Hz}$. The anemometer was placed at a height of $3.7 \mathrm{~m}, 7 \mathrm{~m}$ from the barrier, and $5 \mathrm{~m}$ in from the edge.

The sound power level of the source was determined through measurements in an anechoic chamber at a specified input voltage. This voltage was later kept during the measurements in order to get the same output, and thereby be able to find the sound reduction obtained with the barrier. The 
source spectrum contained rather sharps peaks due to resonances in the flexible hose that were spaced about $200 \mathrm{~Hz}$ apart. In order to avoid this effect, all the measured spectra were averaged over $200 \mathrm{~Hz}$.

\section{Theory}

The spatial and temporal fluctuations in temperature and wind velocity, i.e. the atmospheric turbulence, cause the sound waves to be scattered into acoustic shadow regions and to be decorrelated. Decorrelation effects are usually of interest for line-of-sight propagation.

The model for calculating the influence of the turbulence scattering for barrier situations is based on the model by Daigle [5]. A further developed model [8] uses a small set of precalculated data to predict the scattered power for different geometrical configurations and frequencies. This prediction scheme is here extended to be applicable to a more general barrier shape, and also interference effects due to a ground surface are taken into account.

The sound scattering cross-section used for the scattered power due to the turbulence is a single-scattering approximation where the field incident on a scattering object is assumed to be well approximated by the field calculated for a non-turbulent atmosphere. This means that the model is restricted to situations where the propagation distance is not too large and the fluctuations of the medium not too strong.

The atmospheric turbulence is approximated as homogeneous and isotropic, which means that it is described by the same statistics in all points independent of direction. It is also assumed that the source and receiver are in the far field. This means that they are far away from where the dominant scattering is produced in relation to the largest scale $L_{0}$ of the inhomogeneities of the medium and to the size $L$ of the dominant-scattering volume. It is also assumed that $L \gg \lambda, L \gg L_{0}$, and $R \gg k L L_{0}$ are fulfilled, where $\lambda$ is the acoustic wavelength, $k$ the acoustic wave number, and $R$ the distance from a scattering volume element to the source or to the receiver [6]. Using the correlation length $l$ of the turbulent atmosphere (usually assumed to be around one meter), the last inequality can be relaxed to

$$
R \gg l^{2} / \lambda,
$$

as established in Ref. [7]. (See also Ref. [5].) The last inequality says that the radius of the first Fresnel zone $(R \lambda)^{1 / 2}$ is large compared to $l$, which is a condition for treating the scattering from different points as being uncorrelated. 


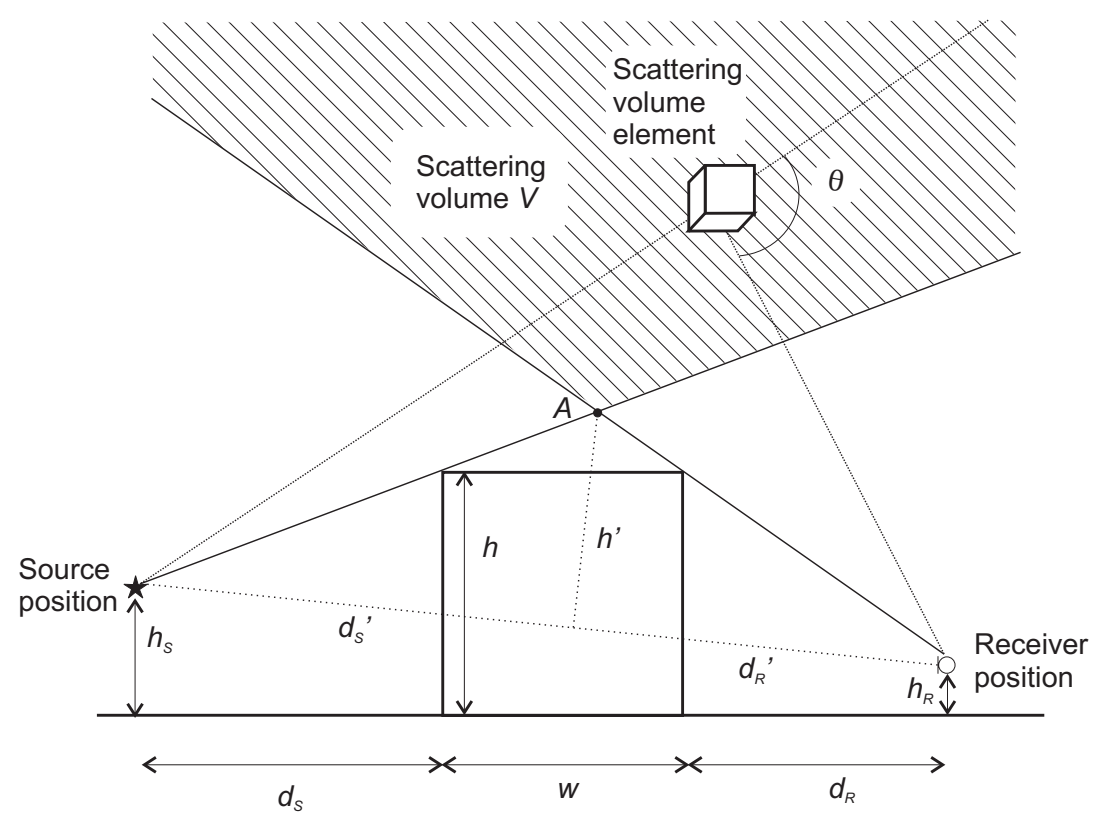

Figure 2. Geometry for the sound scattering cross-section.

Then the time average of the total received scattered power can be written as [5]

$$
W_{s}=\int_{V} p_{0}^{2} \frac{\sigma(\theta)}{\rho^{2}} d V
$$

where $p_{0}$ is the amplitude of the incident pressure, $\sigma(\theta)$ the scattering crosssection, $\theta$ the scattering angle, and $\rho$ the distance from a volume element to the receiver. See Figure 2. The volume of integration $V$ consists of all points in line of sight from both source and receiver (i.e. the striped area in Figure 2).

For the locally homogeneous and isotropic turbulence in the inertial range, following the Kolmogorov spectrum, the structure parameters can be defined from the structure functions

$$
D_{v, T}(x)=C_{v, T}^{2} x^{2 / 3},
$$

where $C_{v}$ and $C_{T}$ are the structure parameters of the wind turbulence and of the temperature turbulence, respectively. 
For the fluctuating velocity $v$ in the direction of $x$ and temperature $T$, and two points, $x_{1}$ and $x$, the structure functions can be defined as

$$
D_{v}(x)=<\left[v\left(x_{1}+x\right)-v\left(x_{1}\right)\right]^{2}>, D_{T}(x)=<\left[T\left(x_{1}+x\right)-T\left(x_{1}\right)\right]^{2}>,
$$

where $<\cdot>$ denotes an ensemble average. The time average can be used instead of the ensemble average when the hypothesis of frozen turbulence is used. For a given scattering angle $\theta$ and acoustic wave number $k$, the wave number $\kappa$ of the turbulence that causes the scattering is found from the Bragg condition

$$
\kappa=2 k \sin \frac{\theta}{2}
$$

For these conditions the scattering cross-section can be written as [6]

$$
\sigma(\theta)=0.03 k^{1 / 3} \frac{\cos ^{2} \theta}{[\sin (\theta / 2)]^{11 / 3}}\left[0.14 \frac{C_{T}^{2}}{T_{0}^{2}}+\frac{C_{v}^{2}}{c_{0}^{2}} \cos ^{2} \frac{\theta}{2}\right],
$$

where $T_{0}$ is the mean temperature in Kelvin and $c_{0}$ the mean sound speed. In Eq. (6) it can be seen that the scattering increases with frequency as $f^{1 / 3}$.

According to this model the scattered power will, relative to free field, change with the same factor as the geometry is scaled. To see this let the height of the screen, as well as its distance from source and receiver, be doubled. Substituting for these new variables in the integral (2) will cause an increase by a factor eight in $d V$ and a factor four in $\rho^{2}$, whereas $p_{0}^{2}$ will stay constant relative to free field. As a result the scattered power will be doubled, i.e. increased by 3 $\mathrm{dB}$, relative to free field.

The turbulence scales larger than in the source range have strengths that are influenced by the large-scale structures of the terrain, and are not easily determined. Since the scattering into barrier shadows has been concluded to influence the mean sound power only at higher frequencies, only the smaller wave numbers of the turbulence are considered (see the Bragg condition (5)). The turbulence spectrum in the source range and in a part of the inertial range is omitted, and $L_{0}=1 \mathrm{~m}$ is assumed instead of a typical value in the order of $100 \mathrm{~m}$. This limits the application of the prediction scheme to high enough frequencies and steep enough geometries. The limitation to steep geometries is in accordance with the other model assumptions because for flatter geometries more power will come via multiple scattering. The dissipation range of the turbulence includes scales smaller than about a few millimetres and therefore will not be of importance in the audio range. 
Concerning the measurements of the turbulence, the structure parameters $C_{v}$ and $C_{T}$ were estimated from the longitudinal turbulence spectrum in the direction of the mean wind. A least square fit in the range $0.5-5 \mathrm{~Hz}$ of the turbulence fluctuations was made of

$$
W_{v, T}(\omega)=\frac{6 \pi}{5} A C_{v, T} U^{2 / 3} \omega^{-5 / 3},
$$

where $A \approx 0.0330, U$ is the mean wind speed, $\omega$ is the angular frequency of the fluctuations, and $W_{v}$ and $W_{T}$ are the power spectra of the velocity and the temperature fluctuations, respectively. (See e.g. [6, 9] for more thorough descriptions of the theory and the measurements of the structure parameters.)

For a ground reflected wave the spherical reflection coefficient [10] is used with the ground impedance being modelled using the Delany/Bazley model [11] and a flow resistivity of $20000 \mathrm{kNm} / \mathrm{s}^{4}$. The diffraction effect for a single edge is modelled by Hadden/Pierce [12] and the two edges on the barrier are then combined using the scheme from Ref. [13]. This procedure gives four ray paths with complex pressures normalized to a free field reference.

\section{Decorrelation}

When the receiver position is not in an acoustic shadow region, the situation is usually referred to as line-of-sight propagation. For line-of-sight propagation in a fluctuating medium the decorrelation causes interference patterns to be less pronounced. (See e.g. Ref. [14].)

For two sources oscillating with the frequency $f$ and contributing with the complex-valued pressure amplitudes $p_{1}$ and $p_{2}$, the time signal of the total pressure in a homogeneous atmosphere can be written

$$
p_{\text {tot }}=p_{1} \mathrm{e}^{\mathrm{j} 2 \pi f t}+p_{2} \mathrm{e}^{\mathrm{j} 2 \pi f t}
$$

where $t$ is time. (The convention $\mathrm{e}^{\mathrm{j} \omega t}$ is used.) For a turbulent atmosphere, the long-term average of the squared absolute value of the total pressure amplitude can be written [15]

$$
\left\langle\left|p_{\text {tot }}\right|^{2}\right\rangle=\left|p_{1}\right|^{2}+\left|p_{2}\right|^{2}+2\left|p_{1} p_{2}\right| \cos \left[\arg \left(\frac{p_{2}}{p_{1}}\right)\right] \Gamma_{12}
$$

where $\Gamma_{12}$ is the mutual coherence function fulfilling $0 \leq \Gamma_{12} \leq 1$. 
For $N$ sources we get [16]

$$
\left\langle\left|p_{\text {tot }}\right|^{2}\right\rangle=\sum_{i=1}^{N}\left|p_{i}\right|^{2}+2 \sum_{i=1}^{N-1} \sum_{j=i+1}^{N}\left|p_{i} p_{j}\right| \cos \left[\arg \left(\frac{p_{j}}{p_{i}}\right)\right] \Gamma_{i j}
$$

For the turbulence model used here, we have [6, 17]

$$
\Gamma=\exp \left[-\frac{3}{8} D\left(\frac{C_{T}^{2}}{T_{0}^{2}}+\frac{22}{3} \frac{C_{v}^{2}}{c_{0}^{2}}\right) k^{2} \rho_{\Gamma}^{5 / 3} L_{\Gamma}\right]
$$

where $D \approx 0.364, \rho_{\Gamma}$ is the transversal separation between the sources and $L_{\Gamma}$ is the longitudinal distance to the receiver. For a direct and a ground reflected wave, the maximum vertical separation between their paths is chosen as the transversal separation [17]. With the heights $h_{S}$ and $h_{R}$ above the ground of the source and the receiver this implies that $\rho_{\Gamma}=2 h_{S} h_{R} /\left(h_{S}+h_{R}\right)$.

The measured values of $C_{v}$ and $C_{T}$ are taken as input when calculating the mutual coherence between direct and ground reflected waves, both for the diffraction and for the scattering. For the diffraction all the paths are taken as going along the top of the barrier; the direct and ground reflected waves from the source meet at the top barrier edge closest to the source, and vice versa on the receiver side. The diffraction can be seen as contributions along four paths, and Eq. (10) is used.

How the decorrelation is applied to the calculation of the scattering is explained in the following subsection.

\section{Prediction scheme}

The Tables A1 and A2 (in Appendix A) with the data for the prediction scheme contain the scattered power at a chosen frequency $f_{0}=2 \mathrm{kHz}$, for different geometries, and for a given unit strength of the velocity fluctuations or of the temperature fluctuations. The data come from a numerical calculation of the integral (2), where the incident pressure $p_{0}$ is calculated without taking into account the reflections from a ground surface, or the field diffracted by the screen. For a complete description of the calculation see Ref. [1] or [2].

The tabulated values are then transformed for other values of frequency or for a scaling of the geometry. If the frequency $f$ is changed, $\frac{10}{3} \log \left(f / f_{0}\right)$ is added, and if the geometrical length scale is changed a factor $s, 10 \log (s)$ is added (i.e. if the distances between source and screen, screen and receiver, and the screen height are all changed with the same factor $s$ ).

When modelling the effect of a ground surface, a good way would be to include the ground reflected wave in $p_{0}$ when the integral (2) is calculated, as well 
as the ground reflection on the receiver side and the decorrelation between direct and ground reflected waves. However, in order to avoid recalculating the integral (2) for each height above the ground of the source and receiver and for each frequency, a point $A$ is chosen, around which the dominating part of the scattering is assumed to be produced. All the direct and ground reflected paths via all scattering volume elements are thereby approximated by the single set of paths that go through $A$. The single set of sound paths from source to receiver via the point $A$ consists of four paths, as seen from mirroring the source and the receiver in the ground surface. If $W_{0}$ is the scattered power when omitting the ground (corresponding to the values in the Tables A1 and A2), then the total power can be approximated as $W_{0} \cdot C_{S} \cdot C_{R}$. Here $C_{S}$ and $C_{R}$ are correction factors, both with values between 0 and 4 . The factors $C_{S}$ and $C_{R}$ are the expected powers relative to free field at $A$ without the barrier, with the ground, and where a decorrelation between direct and ground reflected sound waves is included. $C_{S}$ is calculated from the source and the image source to the point $A$, and $C_{R}$ is to the same point $A$, but from the receiver position. The decorrelation considers the atmospheric turbulence, but for a better prediction it should also take into account ground and terrain properties and a correction that models the dominating scattering as coming from a small volume around the point $A$ rather than only from that point. The last statement is of most importance at high frequencies where the interference pattern has room to vary significantly inside the volume where the dominating scattering is produced.

The point $A$ is chosen to be the lowest point in line of sight from both the source and the receiver. For a thin screen, $A$ is at the screen edge. (See Figure 2.) This choice of the point $A$ is motivated since the scattering cross-section (6) in general becomes large when the scattering angle becomes small. If $\theta=90^{\circ}$ at $A$, however, there is no scattering, and another choice of $A$ would be motivated. Moreover, for $A$ very close to the source and far away from the receiver (or vice versa) the scattered power from $A$ will be low due to the large difference in the distances (see Eq. (2)).

The presented prediction scheme is applied in the following for the comparison with the measured data.

\section{Comparison between predictions and measurements}

Measurements of the sound reduction obtained with a thick barrier were performed simultaneously with turbulence measurements on two different days.

The results for six different geometries are presented in Figures 3-8. The distance from the barrier to the receiver is varied $\left(d_{r}=4,8\right.$, and $\left.18 \mathrm{~m}\right)$ and the 
heights of the source and the receiver are changed. The situation with a high source position $(1.25 \mathrm{~m})$ and the receiver on the ground is exchanged for a high receiver position $(1.25 \mathrm{~m})$ and the source on the ground. When the source is on the ground a source height $h_{S}$ of around $3 \mathrm{~cm}$ is used as input in the model (see each figure caption for the exact value used for $h_{S}$ ). For the receiver on the ground the height $h_{R}$ is taken to be $3 \mathrm{~mm}$.

The results to the left are from the calmer situations, and to the right are those from the more windy situations. The measured values of the structure parameters are shown in each figure caption.

In the calculated results the air absorption is taken into account using ISO 9613-1:1993, based on the mean temperature that was about $19^{\circ} \mathrm{C}$ and the relative humidity that was about 50\% during the measurements. The atmospheric pressure was assumed to be $1013 \mathrm{hPa}$.

In the plots the measured results are represented by a thick solid line and the total predictions, including diffraction, decorrelation and scattering, are represented by a thick dashed line. The thin dash-dotted line represents the turbulence scattering only and the thin solid line represents the diffraction only, without considering the decorrelation.

At frequencies lower than about $2 \mathrm{kHz}$ the results show little influence of turbulence scattering. The measurements are in fairly good agreement with the calculations in this range. It does, however, look like an offset in some situations. These offsets may be due to refraction caused by the mean wind speed profile. For the downwind situations (right) the measured values are mainly higher than the predictions and for the upwind situations (left) the measured values are mainly lower than the predictions.

For frequencies above $2 \mathrm{kHz}$ strong scattering effects, up to $10 \mathrm{~dB}$, are shown in the more windy and turbulent situations, especially for the longer receiver distances. For these high-frequency data the agreement between the predictions and the measurements is fairly good. The model does, however, seem to overpredict the scattered power for the highest frequencies, i.e. above $6-8 \mathrm{kHz}$. This may partly be the error due to the single-scattering approximation, i.e. considering multiple scattering could mean a change in the incident pressure wave $p_{0}$ in Eq. (2).

The decorrelation in the predictions seems in general to be stronger than in the measurements. This can be seen for example in Figure 5 (right), where the prediction shows no interference effects above $2 \mathrm{kHz}$, whereas the measurements do, up to about $3 \mathrm{kHz}$. This is probably due to that the strength of the turbulence is measured above the barrier and that the main part of the medium 

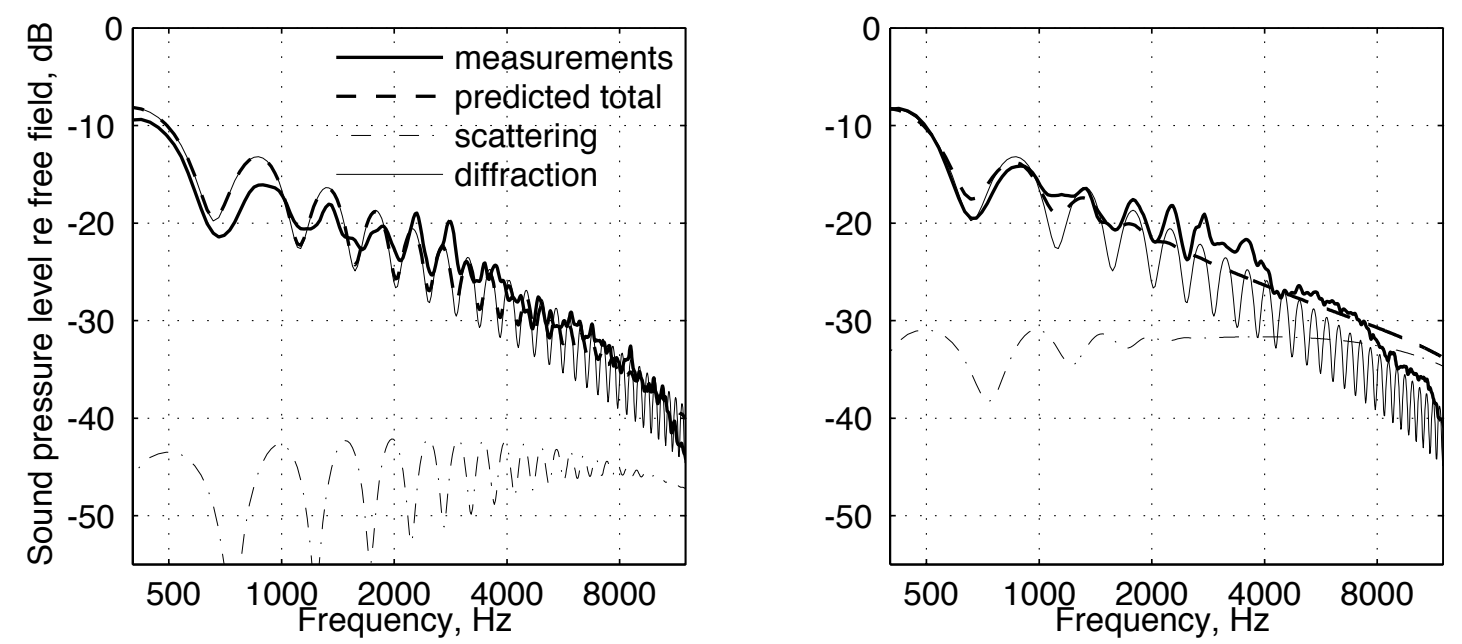

Figure 3. Measurement and prediction for $h_{S}=1.25 \mathrm{~m}, h_{R}=0.3 \mathrm{~cm}$, and $d_{R}=4 \mathrm{~m}$. To the left $C_{v}^{2}=0.4 \mathrm{~m}^{4 / 3} / \mathrm{s}^{2}$ and $C_{T}^{2}=0.2^{\circ} \mathrm{K} / \mathrm{m}^{2 / 3}$; to the right $C_{v}^{2}=8 \mathrm{~m}^{4 / 3} / \mathrm{s}^{2}$ and $C_{T}^{2}=3.5^{\circ} \mathrm{K} / \mathrm{m}^{2 / 3}$. (The same units are used in the following Figures.)

that influences the decorrelation is closer to ground, and there the turbulence may be weaker.

For the low source position the field in the neighborhood of the barrier top experiences an interference minimum at high frequencies, around $10 \mathrm{kHz}$ (see Figures 6-8). In the model predictions, this minimum leads to a larger interference dip than is shown by the measurements. If the interference is included in $p_{0}$ when the integral (2) is calculated, the interference dip would be much less pronounced since at these high frequencies, the volume from which the dominating scattering comes will not be occupied by a field described mainly by destructive interference. Therefore, the decorrelation should increase faster at higher frequencies in order to compensate for the spatially narrower interference pattern compared to the size of the dominant-scattering volume. This problem appears not only for the scattering. It could be seen as a general difficulty at high frequencies for most acoustic prediction methods. Also the source and the boundary will contain details that are difficult to describe, but are important at high frequencies. For example when the wavelength is about the size of the source, the point source model may be a poor representation. The usual approach to handle this would be to model the details in a statistical way in a wide-band analysis. 

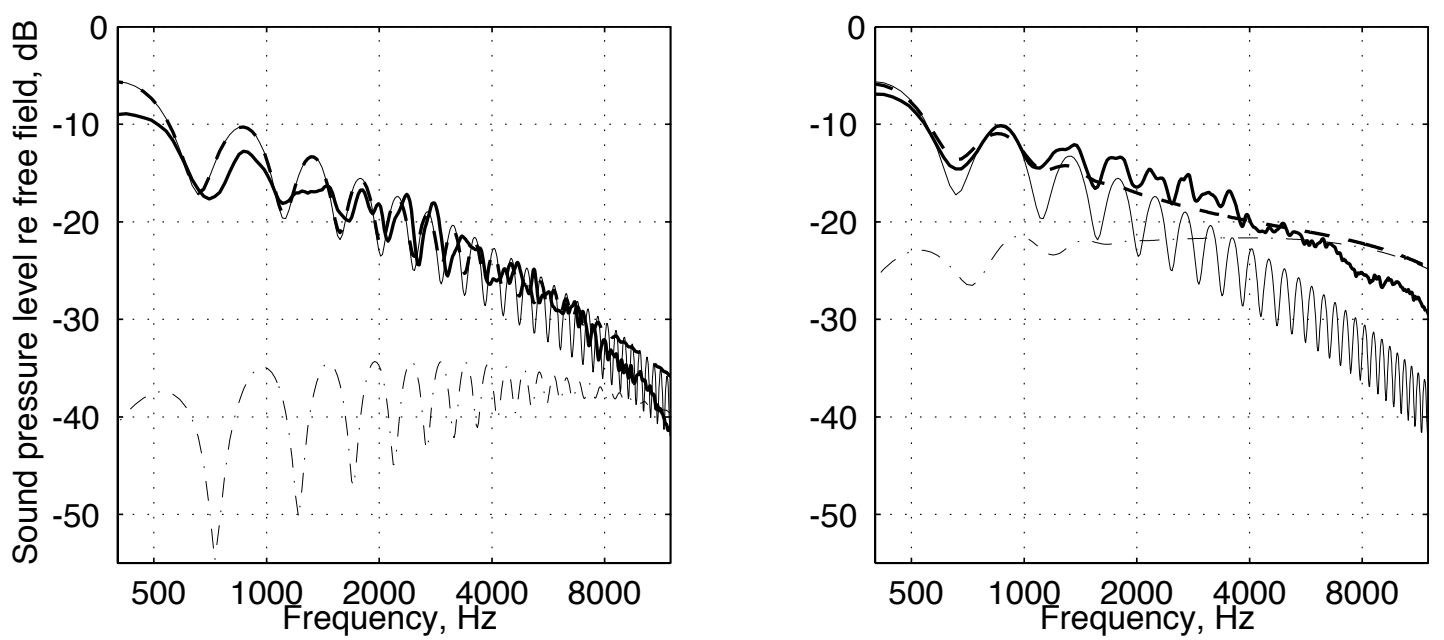

Figure 4. Measurement and prediction for $h_{S}=1.25 \mathrm{~m}, h_{R}=0.3 \mathrm{~cm}$, and $d_{R}=8 \mathrm{~m}$. To the left $C_{v}^{2}=0.4$ and $C_{T}^{2}=0.3$; to the right $C_{v}^{2}=15$ and $C_{T}^{2}=4$.
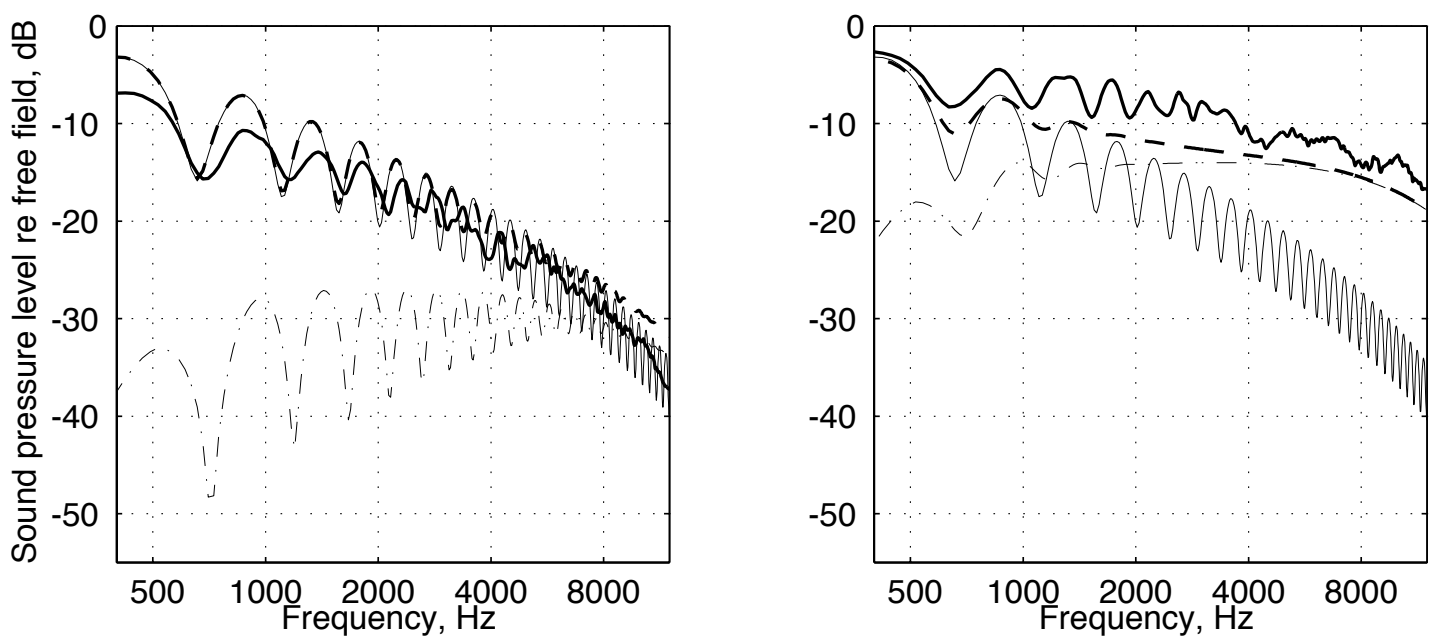

Figure 5. Measurement and prediction for $h_{S}=1.25 \mathrm{~m}, h_{R}=0.3 \mathrm{~cm}$, and $d_{R}=18 \mathrm{~m}$. To the left $C_{v}^{2}=0.4$ and $C_{T}^{2}=0.2$; to the right $C_{v}^{2}=15$ and $C_{T}^{2}=3.5$. 

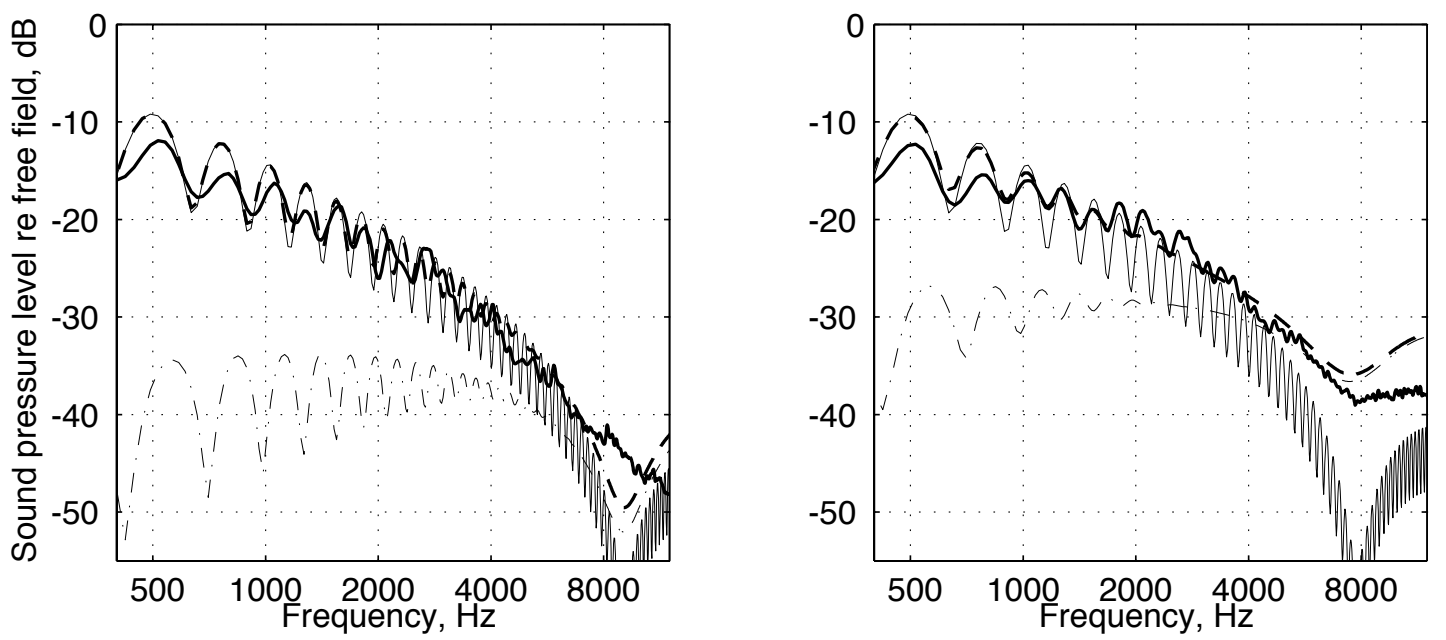

Figure 6. Measurement and prediction for $h_{S}=2.9 \mathrm{~cm}$ (left), and $3.4 \mathrm{~cm}$ (right), $h_{R}=$ $1.25 \mathrm{~m}$, and $d_{R}=4 \mathrm{~m}$. To the left $C_{v}^{2}=2$ and $C_{T}^{2}=1$; to the right $C_{v}^{2}=15$ and $C_{T}^{2}=2$.
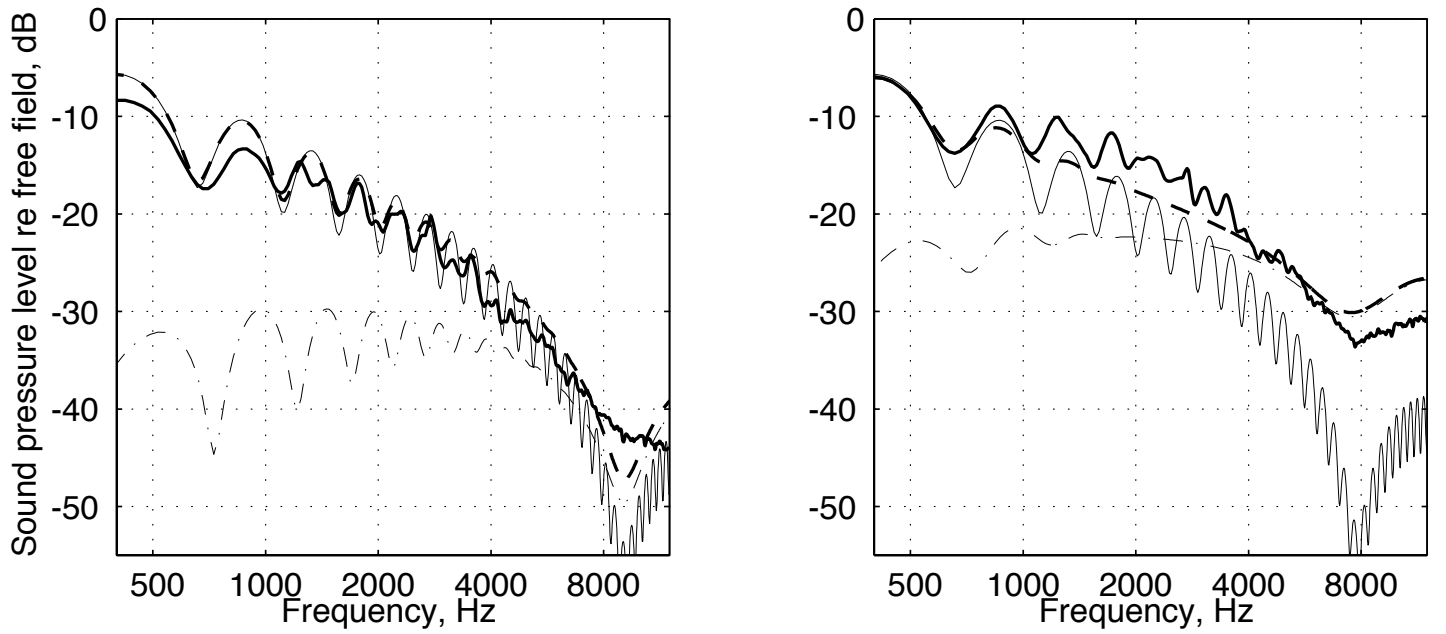

Figure 7. Measurement and prediction for $h_{S}=2.9 \mathrm{~cm}$ (left), and $3.4 \mathrm{~cm}$ (right), $h_{R}=$ $1.25 \mathrm{~m}$, and $d_{R}=8 \mathrm{~m}$. To the left $C_{v}^{2}=1.5$ and $C_{T}^{2}=0.8$; to the right $C_{v}^{2}=17$ and $C_{T}^{2}=2$. 

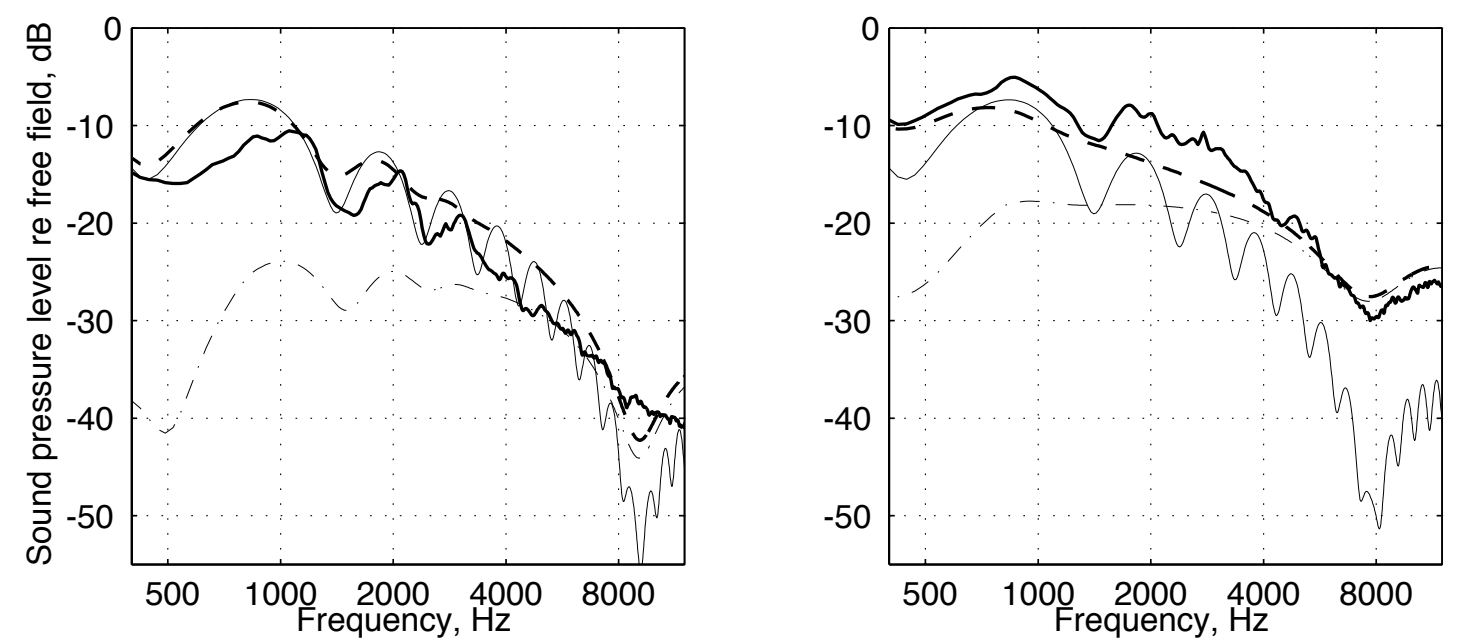

Figure 8. Measurement and prediction for $h_{S}=2.9 \mathrm{~cm}$ (left), and $3.4 \mathrm{~cm}$ (right), $h_{R}=$ $1.25 \mathrm{~m}$, and $d_{R}=18 \mathrm{~m}$. To the left $C_{v}^{2}=2$ and $C_{T}^{2}=1$; to the right $C_{v}^{2}=14$ and $C_{T}^{2}=2$.

\section{Conclusions}

Looking at the overall results for both the calm and for the windy situations, the predictions agree fairly well with the measurements.

The influence of the turbulence scattering on the sound reduction obtained with a barrier increases when the frequency is increased or when the geometry is increased in scale (as shown in comparison to earlier measurements $[5,18,8]$ ).

The scattering due to turbulence is shown to be able to significantly increase the noise level behind a barrier. The increase shown here is up to about $5 \mathrm{~dB}$ at $4 \mathrm{kHz}$ and up to about $10 \mathrm{~dB}$ at $8 \mathrm{kHz}$. For larger geometries even stronger effects are assumed to be possible.

The model includes many approximations and conditions that are not always fulfilled. For instance, assuming that the turbulence is homogeneous, so that it needs to be measured in one point only, is probably a crude approximation. Moreover, the condition (1) that motivates the volume integration of the scattered power is not met at the higher frequencies. Also, neglecting the diffracted field in the wave irradiating the scattering volume ( $p_{0}$ in Eq. (2)) could cause a significant error. This problem is connected to the approximation that the scattered and the diffracted fields are uncorrelated. Moreover, the error due to the single-scattering approximation might grow large at the higher frequencies, where the scattering cross-section is larger, which may cause the overprediction in the model at the highest frequencies, here above about 6- 
$8 \mathrm{kHz}$. The fairly good agreement between the predictions and the measurements does, however, indicate that the model is applicable.

For future work a model similar to the one used here can be developed to take into account barriers of finite length, a non-constant sound speed profile, and inhomogeneous and anisotropic turbulence.

\section{Acknowledgements}

The authors wish to thank Wolfgang Kropp for fruitful discussions and critical reading. This work was financially supported by MISTRA (Swedish Foundation for Strategic Environmental Research). 


\section{Appendix A}

The Tables A1 and A2 contain the scattered power, in $\mathrm{dB}$ relative to free field, calculated for the frequency $f_{0}=2 \mathrm{kHz}$, without the influence of a ground. In Table A1, $C_{v}^{2}=1 \mathrm{~m}^{4 / 3} / \mathrm{s}^{2}$ and $C_{T}^{2}=0$. In Table A2, $C_{v}^{2}=0$ and $C_{T}^{2}=1^{\circ} \mathrm{K} / \mathrm{m}^{2 / 3}$. The screen height $h^{\prime}$ is the height of the equivalent thin screen, which has the edge at the point $A$. The distance $d_{R}^{\prime}$ between screen and receiver is varied as well as $h^{\prime}$. The distance $d_{S}^{\prime}=40 \mathrm{~m}$ between source and screen is kept constant. (See Figure 2.) The scattered power for other frequencies and geometries is found using transformation properties, as described in the paper.

\begin{tabular}{rcccccccccc} 
& $d_{R}^{\prime}=10$ & 20 & 30 & 40 & 50 & 60 & 70 & 80 & 90 & 100 \\
\hline$h^{\prime}=5$ & -41.6 & -33.9 & -30.2 & -27.9 & -26.2 & -24.9 & -23.9 & -23.1 & -22.4 & -21.8 \\
10 & -49.9 & -44.0 & -39.5 & -36.7 & -34.7 & -33.1 & -31.8 & -30.9 & -30.1 & -29.3 \\
15 & -52.1 & -48.9 & -45.8 & -42.9 & -40.7 & -39.1 & -37.7 & -36.6 & -35.5 & -34.7 \\
20 & -53.8 & -51.0 & -48.8 & -46.8 & -45.0 & -43.5 & -42.1 & -40.9 & -39.9 & -39.1 \\
25 & -55.4 & -52.4 & -50.4 & -48.8 & -47.5 & -46.2 & -45.1 & -44.0 & -43.1 & -42.2 \\
30 & -57.0 & -53.8 & -51.5 & -50.6 & -48.9 & -47.8 & -46.8 & -45.9 & -45.2 & -44.4 \\
35 & -58.6 & -55.1 & -52.7 & -51.1 & -49.8 & -48.8 & -47.9 & -47.1 & -46.4 & -45.7 \\
40 & -59.9 & -56.5 & -53.9 & -52.1 & -50.7 & -49.6 & -48.7 & -48.0 & -47.3 & -46.6
\end{tabular}

Table A1. Scattered level due to a unit strength of velocity turbulence for different values of the screen height $h^{\prime}$ and the screen-receiver distance $d_{R}^{\prime \prime}$ (both in meters).

\begin{tabular}{rcccccccccc} 
& $d_{R}^{\prime}=10$ & 20 & 30 & 40 & 50 & 60 & 70 & 80 & 90 & 100 \\
\hline$h^{\prime}=5$ & -44.0 & -39.1 & -36.0 & -34.0 & -32.5 & -31.3 & -30.4 & -29.6 & -28.9 & -28.3 \\
10 & -47.4 & -44.7 & -42.4 & -40.5 & -39.1 & -37.9 & -36.9 & -36.0 & -35.3 & -34.7 \\
15 & -48.9 & -46.7 & -45.1 & -43.6 & -42.4 & -41.4 & -40.5 & -39.7 & -39.0 & -38.4 \\
20 & -50.2 & -48.0 & -46.4 & -45.2 & -44.1 & -43.2 & -42.4 & -41.7 & -41.1 & -40.5 \\
25 & -51.4 & -49.0 & -47.4 & -46.2 & -45.2 & -44.3 & -43.6 & -42.9 & -42.3 & -41.8 \\
30 & -52.5 & -50.0 & -48.3 & -47.0 & -46.0 & -45.1 & -44.4 & -43.7 & -43.2 & -42.6 \\
35 & -53.6 & -51.0 & -49.2 & -47.8 & -46.7 & -45.8 & -45.0 & -44.4 & -43.8 & -43.3 \\
40 & -54.6 & -52.0 & -50.0 & -48.5 & -47.4 & -46.4 & -45.6 & -44.9 & -44.3 & -43.8
\end{tabular}

Table A2. Scattered level due to a unit strength of temperature turbulence for different values of the screen height $h^{\prime}$ and the screen-receiver distance $d_{R}^{\prime \prime}$ (both in meters). 


\section{References}

[1] Forssén J. Influence of atmospheric turbulence on sound reduction by a thin, hard screen: A parameter study using the sound scattering crosssection. Proc. 8th Int. Symp. on Long-Range Sound Propagation, The Pennsylvania State University, 1998, pp. 352-64.

[2] Forssén J. Calculation of sound reduction by a screen in a turbulent atmosphere. Report F 98 - 01, Chalmers University of Technology, Göteborg, Sweden (ISSN 0283 - 832X), 1998.

[3] Forssén J. Calculation of sound reduction by a screen in a turbulent atmosphere using the parabolic equation method. Acustica 1998, Vol. 84, pp. 599-606.

[4] Forssén J. Calculation of noise barrier performance in a turbulent atmosphere by using substitute sources above the barrier. Acustica 2000, Vol. 86, pp. $269-75$.

[5] Daigle GA. Diffraction of sound by a noise barrier in the presence of atmospheric turbulence. J Acoust Soc Am 1982, Vol. 71, pp. 847-54.

[6] Ostashev VE. Acoustics in moving inhomogeneous media. E \& FN Spon (an imprint of Thomson Professional), London, 1997.

[7] Tatarskii VI The effects of the turbulent atmosphere on wave propagation. Keter Press, Jerusalem, 1971.

[8] Forssén J, Ögren M. Approximative model for the influence of atmospheric turbulence on sound reduction by a thin screen. Proc. 6th International Congress on Sound and Vibration, Technical University of Denmark, Lyngby, 1999, pp. 713-20.

[9] Panofsky HA, Dutton JA. Atmospheric turbulence. John Wiley \& sons, New York, 1984.

[10] Chein CF, Soroka WW Sound propagation along an impedance plane Journal of Sound and Vibration 1975, Vol. 43, pp. 9-20.

[11] Delany ME, Bazley EN Acoustical properties of fibrous absorbent materials Applied Acoustics 1970, Vol. 3, pp. 309-322.

[12] Hadden JW, Pierce AD Sound diffraction around screens and wedges for arbitrary point source locations J. Acoust. Soc. Am 1981, Vol. 69, pp. 12661276.

[13] Salomons EM Sound Propagation in Complex Outdoor Situations with a Non-Refracting Atmosphere: Model Based on Analytical Solutions for Diffraction and Reflection Acustica / Acta Acustica 1997, Vol. 83, pp. 436454. 
[14] Chevret P, Blanc-Benon Ph, Juvé D A numerical model for sound propagation through a turbulent atmosphere near the ground. J Acoust Soc Am 1996, Vol. 100, pp. 3587-99.

[15] Clifford SF, Lataitis RJ. Turbulence effects on acoustic wave propagation over a smooth surface. J Acoust Soc Am 1983, Vol. 73, pp. 1545-50.

[16] L'Espérence A, Herzog P, Daigle GA, Nicolas JR. Heuristic model for outdoor sound propagation based on an extension of the geometrical ray theory in the case of a linear sound speed profile. Applied Acoustics 1992, Vol. 37, pp. 111-39.

[17] Ostashev V, Clifford S, Lataitis R, Blanc-Benon P, Juve D. The effects of atmospheric turbulence on the interference of the direct and ground reflected waves. Proc. 29th Inter-Noise, Nice, 2000, pp. 217-222.

[18] Ögren M, Forssén J. Measurements of sound reduction by a noise barrier in the presence of wind and atmospheric turbulence. Proc. 6th International Congress on Sound and Vibration, Technical University of Denmark, Lyngby, 1999, pp. 721-26. 